\title{
100 milliards de rayons cosmiques détectés dans l'expérience AMS-02
}

Sandy Aupetit ${ }^{(1)}$ (sandy.aupetit@gmail.com), David Maurin(1) (dmaurin@lpsc.in2p3.fr) et Vincent Poireau ${ }^{(2)}$ (vincent.poireau@lapp.in2p3.fr)

(1) LPSC, Université Grenoble Alpes, CNRS/IN2P3, 53 avenue des Martyrs, 38026 Grenoble (2) LAPP, Université Savoie Mont Blanc, CNRS/IN2P3, 9 chemin de Bellevue, 74941 Annecy

\section{Notre planète est bombardée}

en permanence par le rayonnement cosmique, un ensemble

de particules en provenance

de l'espace.

\section{Orbitant à 400 km au-dessus}

de la Terre depuis la station

spatiale internationale,

l'expérience AMS-02 a déjà détecté

plus de 100 milliards de particules

depuis son installation,

le 19 mai 2011.

Ces données, d'une précision

inégalée, permettent aujourd'hui

des avancées majeures

en astrophysique, et apportent

de précieux indices dans

la recherche de la mystérieuse

matière noire.
Depuis leur découverte il y a plus d'un siècle, les rayons cosmiques [1,2] n'ont cessé de susciter l'intérêt de la communauté scientifique, et ont été à l'origine de nombreuses avancées en physique des particules et en astrophysique. Ce rayonnement arrivant au sommet de l'atmosphère terrestre, est constitué à la fois de particules chargées et de particules neutres. On trouve dans la composante chargée essentiellement des protons et des noyaux d'hélium (88\% et $10 \%$ respectivement), puis des noyaux d'atomes plus lourds (1\%), des électrons $(1 \%)$, et une infime portion d'antimatière avec des positons (ou anti-électrons) et des antiprotons. La composante neutre est, quant à elle, constituée de photons de haute énergie et de neutrinos.

Une caractéristique étonnante du spectre des rayons cosmiques reçus sur Terre (fig. 1) est qu'il s'étend sur près de douze ordres de grandeur en énergie, et trente ordres de grandeur en flux ! Les énergies en jeu peuvent ainsi être colossales, et beaucoup plus grandes que celles que l'on peut produire en laboratoire sur accélérateur, avec par exemple le LHC. Le flux décroît par ailleurs très rapidement avec l'énergie pour atteindre seulement une particule par $\mathrm{km}^{2}$ et par siècle à $10^{20} \mathrm{eV}$ ! Les rayons cosmiques de très basses énergies, les plus nombreux, proviennent essentiellement du Soleil. Aux énergies intermédiaires, entre typiquement $10^{8}$ et $10^{16} \mathrm{eV}$, ils sont issus de notre Galaxie, en provenance des supernovæ ou des pulsars. Les rayons cosmiques d'ultra-hautes énergies sont vraisemblablement d'origine extragalactique, majoritairement produits et accélérés dans les noyaux actifs de galaxies.
Heureusement pour la vie sur Terre, l'atmosphère atténue fortement la radiation reçue au sol. Les interactions avec les atomes de l'air donnent lieu à des cascades de particules moins énergétiques, qui sont mises à profit pour étudier les propriétés du rayonnement cosmique aux plus hautes énergies. C'est le cas par exemple des expériences H.E.S.S. en Namibie [3] et Auger en Argentine [4]. Mais pour étudier les rayons cosmiques provenant directement du cosmos, il est nécessaire de se placer au-dessus de l'atmosphère, afin d'éviter toute interaction. C'est pourquoi, depuis 1950, des détecteurs sont embarqués sur ballon (par exemple CREAM), ou mieux encore, envoyés dans l'espace (AMS, Fermi, PAMELA).

\section{L'expérience AMS-02 sur la station spatiale internationale}

La collaboration internationale AMS (en anglais Alpha Magnetic Spectrometer) est constituée de plusieurs centaines de physiciens et ingénieurs. Elle est dirigée depuis ses débuts par Samuel Ting, prix Nobel de physique en 1976 pour la découverte du méson J/ $\psi$. Le 19 mai 2011, le détecteur AMS-02 a été installé à $400 \mathrm{~km}$ d'altitude sur la plus grande structure existant dans l'espace, de la taille d'un terrain de football, la station spatiale internationale (ISS). Le lancement a été effectué grâce à la navette spatiale américaine Endeavour, depuis la base de Cap Canaveral. Depuis son installation sur l'ISS (fig. 2), AMS-02 effectue une orbite autour de la Terre toutes les 90 minutes et accumule des données en continu tous les jours de l'année. 


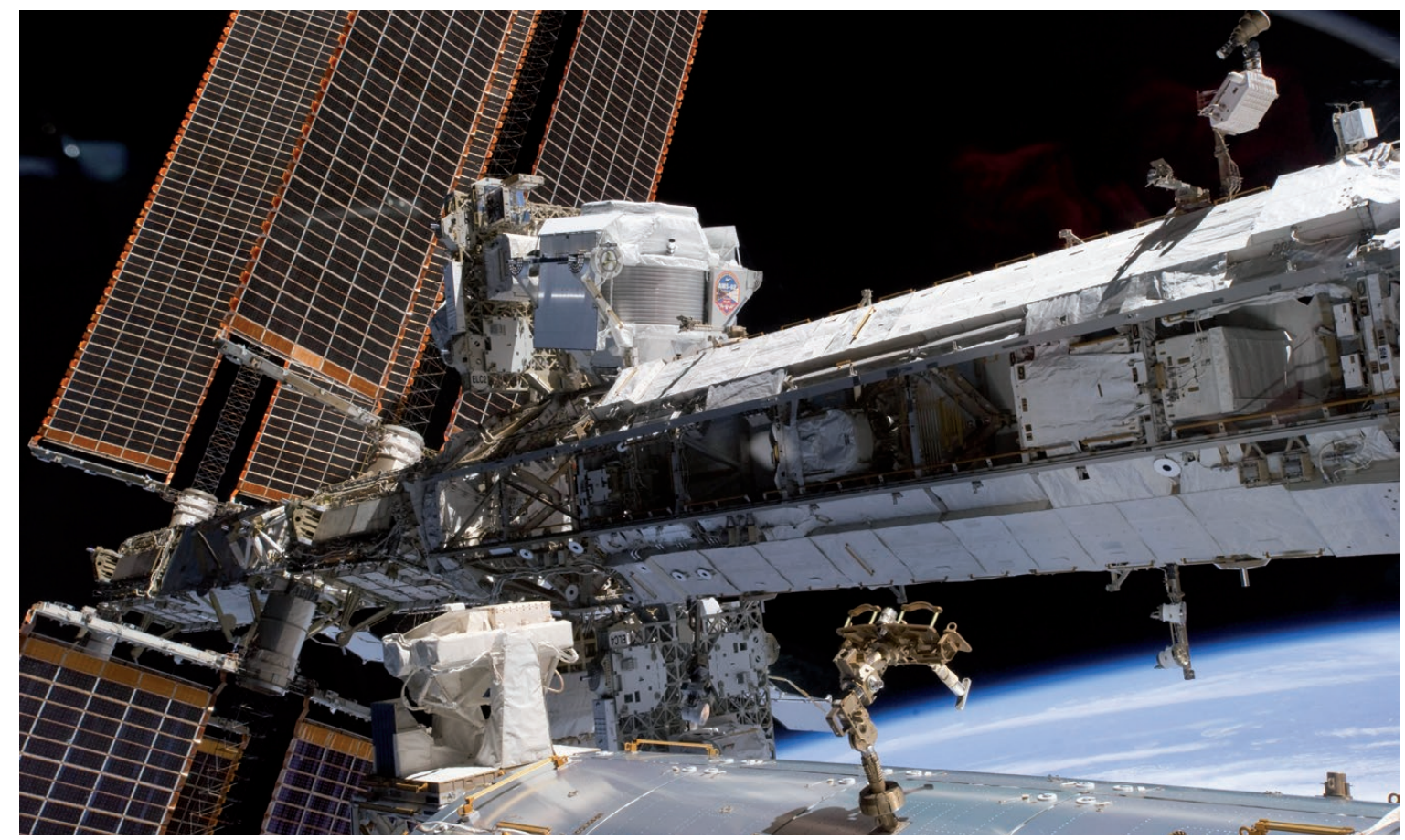

2. AMS-02 sur I'ISS. Vue du détecteur AMS-02 sur la poutre centrale de la station spatiale internationale. ๑ NASA

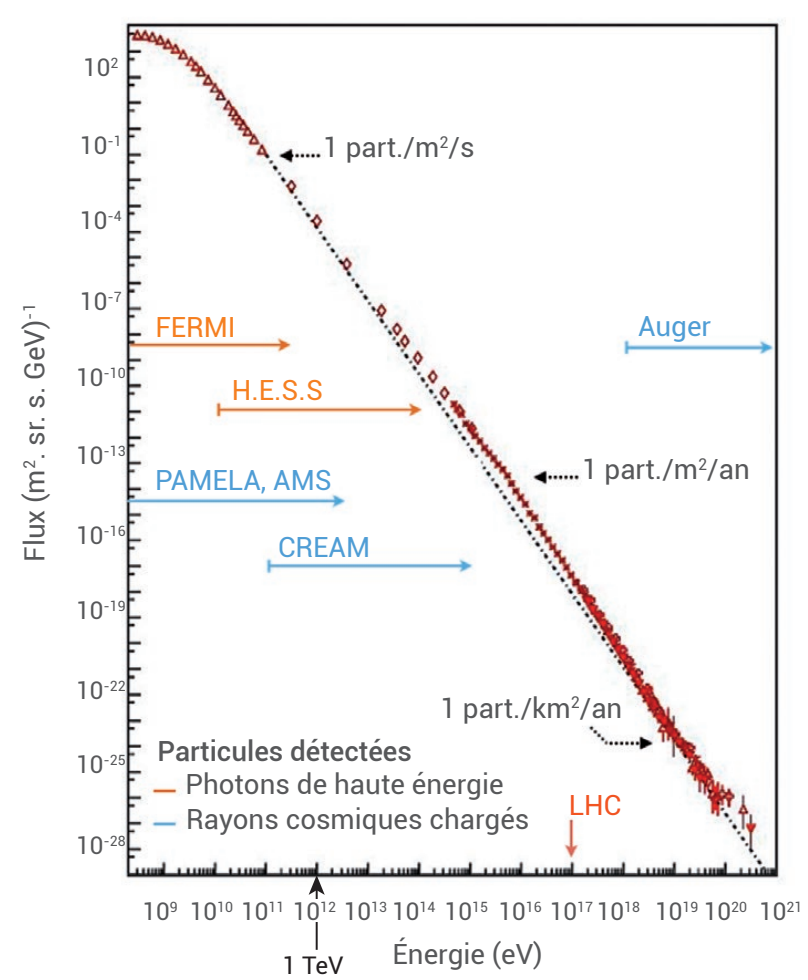

1. Spectre du rayonnement cosmique. Cette figure représente le flux de rayons cosmiques chargés (c'est-à-dire le nombre de particules par mètre carré, par stéradian, par seconde et par gigaélectronvolt) reçu au sommet de l'atmosphère terrestre, en fonction de leur énergie totale. Cette mesure a été obtenue en combinant les résultats de plusieurs expériences : des ballons, des satellites et des réseaux de détecteurs au sol. Pour repère, l'intervalle en énergie couvert par plusieurs expériences est annoté. À titre de comparaison, le plus puissant accélérateur de particules sur terre, le LHC au CERN, délivre une énergie qui correspondrait à un rayon cosmique de $10^{17} \mathrm{eV}$ interagissant avec un noyau de l'atmosphère.

\section{Le détecteur AMS-02}

Capable de détecter des particules de $500 \mathrm{MeV}$ à plusieurs $\mathrm{TeV}$, l'expérience enregistre entre 200 rayons cosmiques par seconde à proximité de l'équateur et 2000 près des pôles. (Cette différence est liée au champ magnétique dipolaire terrestre qui dévie les trajectoires des particules de basses énergies : cet effet est plus efficace à l'équateur que près des pôles magnétiques.) Cela correspond en moyenne à 40 millions d'événements par jour, représentant près de 100 Go de données qu'il faut transférer sur Terre et traiter afin de reconstruire la trajectoire, l'énergie et l'identité des particules. Après six ans de fonctionnement, AMS-02 affiche au compteur plus de 100 milliards d'événements, ce qui est supérieur à l'ensemble des données récoltées depuis la découverte du rayonnement cosmique en 1912 ! Les principaux objectifs scientifiques d'AMS sont la mesure précise des flux des différentes espèces de rayons cosmiques et la recherche indirecte de matière noire, qui sont discutés plus bas. Le principe de détection est détaillé dans l'encadré p. 28. Le détecteur AMS-02 a, en particulier, un atout incontestable, avec son aimant qui permet de distinguer les particules de matière et d'antimatière (comme les électrons et positons); il restera une référence pour de très nombreuses années. 
\>

\section{Les autres expériences \\ dans 1'espace}

L'expérience de référence qui a précédé AMS-02 est le satellite PAMELA, lancé en 2006 sur une orbite de $580 \mathrm{~km}$ d'altitude. Cependant, sa plus petite taille lui donne un pouvoir de collecte 100 fois plus faible, conduisant à des données moins précises et moins étendues en énergie qu'AMS-02. Le satellite Fermi lancé, quant à lui, en 2008 à une altitude de $560 \mathrm{~km}$, observe les rayons gamma de haute énergie, et étudie donc la partie neutre du rayonnement cosmique. Plus récemment, on trouve le détecteur CALET, installé sur l'ISS en août 2015, le satellite DAMPE, mis en orbite en décembre 2015, et le détecteur ISS-CREAM placé également sur l'ISS en août 2017. Ces nouvelles expériences ont pour objectif d'étendre les mesures d'AMS-02 jusqu'à $1000 \mathrm{TeV}$ (soit $10^{15} \mathrm{eV}$ ).

\section{Le transport des rayons cosmiques : un voyage peu ordinaire}

\section{Embarquement immédiat : destination la Terre!}

La Voie lactée est une galaxie spirale qui contient une centaine de milliards d'étoiles. En moyenne, quelques étoiles par siècle terminent leur vie en supernovæ, dont l'onde de choc accélère noyaux et électrons pour former le rayonnement cosmique dit galactique, d'énergie comprise entre $10^{8}$ et $10^{16} \mathrm{eV}[1,2]$. À ces énergies, les noyaux sont complètement ionisés, et donc sensibles aux interactions électromagnétiques, tout comme les électrons. D'une part, les trajectoires sont déterminées par la structure complexe du champ magnétique galactique, qui contient une composante régulière mais aussi une composante turbulente de même intensité $(\sim 1 \mu \mathrm{G})$. Ce transport est décrit en première approximation par une équation de type diffusion, équivalente à une marche aléatoire des particules chargées dans la Galaxie. Par ailleurs, les noyaux perdent ou redistribuent leur énergie tout au long de leur parcours, et changent parfois même de nature, au gré des interactions nucléaires avec le milieu interstellaire (constitué de protons et de noyaux d'hélium).

Après un voyage de plusieurs dizaines de millions d'années, dans un environnement s'étendant sur quelques dizaines de milliers d'années-lumière, certains des rayons cosmiques atteignent la zone d'influence du Soleil, appelée cavité solaire. Ils subissent alors l'effet du vent solaire, qui se traduit dans les observations expérimentales par une modulation des flux de particules à basse énergie, variant périodiquement selon l'activité solaire. Après encore une année à vagabonder, ils arrivent aux abords de la Terre et peuvent alors être détectés par une expérience telle qu'AMS-02.

\section{Une brisure inattendue} dans le spectre des noyaux

Une fois les données récoltées par le détecteur, comment remonter la piste des rayons cosmiques ? Comme nous l'avons $\mathrm{vu}$ en introduction, les protons et les noyaux d'hélium sont les particules les plus abondantes du rayonnement cosmique, et ce sont donc les premières espèces qui ont été étudiées en profondeur par la collaboration AMS. Dans le paradigme standard, le spectre des espèces primaires de rayons

\section{Un détecteur de physique des particules dans l'espace}

L'expérience AMS-02 est un détecteur de particules (fig. E1), de conception similaire à ceux que l'on trouve sur un accélérateur, mais limité à la fois en dimensions et en poids pour pouvoir être envoyé dans l'espace. Sa taille est ainsi de $5 \times 4 \times 3$ mètres pour une charge de 7,5 tonnes. En comparaison, le détecteur ATLAS au LHC mesure $25 \times 25 \times 46$ mètres pour 7000 tonnes.

Lorsqu'un rayon cosmique traverse AMS-02, il rencontre plusieurs sous-détecteurs qui vont permettre de mesurer ses caractéristiques. Tous les sous-détecteurs participent de manière redondante à identifier la particule. Ainsi, le détecteur à rayonnement de transition identifie les électrons et les positons (pas de signal laissé par les noyaux). La traversée des compteurs de temps de vol permet de déterminer la direction, la vélocité et la charge de la particule. Au cœur d'AMS-02, le trajectographe mesure la trajectoire de la particule dans le champ magnétique de l'aimant. Le rayon de courbure donne une mesure de la rigidité $\mathrm{R}=\mathrm{pc} / \mathrm{Ze}$ de la particule, exprimée en gigavolts GV (où $p$ est l'impulsion, $Z$ la charge électrique, $c$ la vitesse de la lumière et e la charge élémentaire), et permet de déterminer s'il s'agit d'une particule de matière ou d'antimatière (des charges opposées ont des courbures opposées). Le détecteur Cherenkov fournit lui aussi une estimation de la charge et de l'énergie, alors que le calorimètre électromagnétique mesure l'énergie et la direction des électrons et positons, et les distingue des protons et autres noyaux.

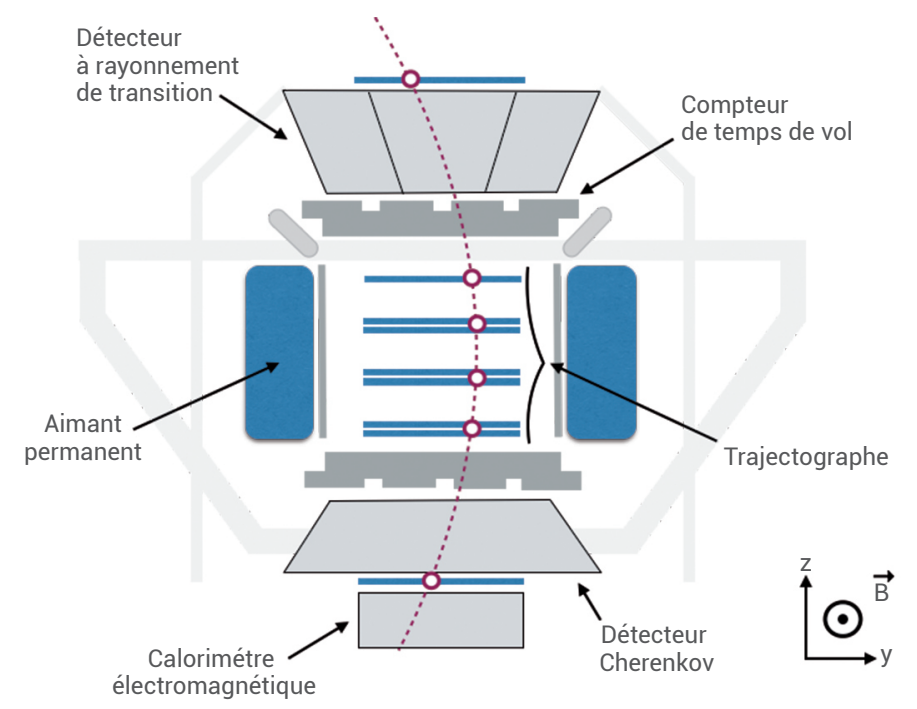

E1. L'expérience AMS-02. 


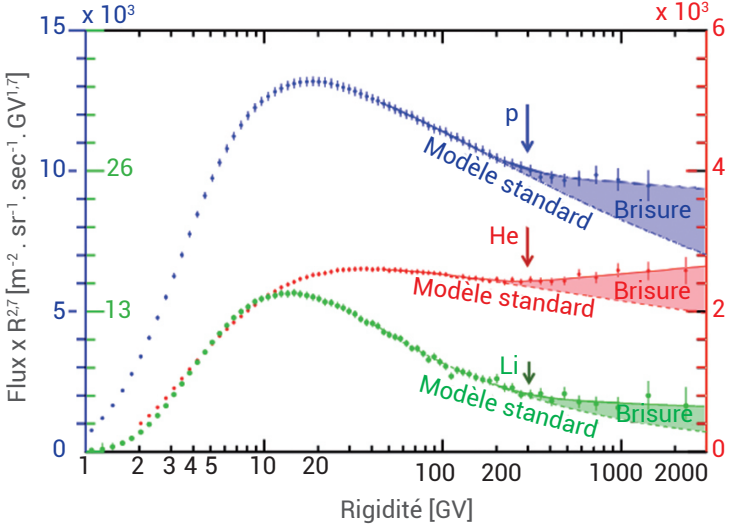

3. Brisure des spectres de p, He et Li. Spectres en protons, noyaux d'hélium et de lithium (résultats préliminaires) mesurés par AMS-02 en fonction de la rigidité. Grâce à sa sensibilité et à la qualité de reconstruction des événements, les barres d'erreurs sont quasiment invisibles $(\sim 3 \%)$ sauf à haute rigidité. Les flèches et les zones pleines indiquent un changement de régime inattendu à environ $300 \mathrm{GV}$ (spectres multipliés par $\mathrm{R}^{2,7}$ pour faire apparaître plus clairement les changements de pente).

cosmiques (c'est-à-dire ceux provenant directement des supernovæ) est en bonne approximation une loi de puissance au-delà de quelques dizaines de $\mathrm{GeV}$ (fig. 1). Cette loi de puissance peut s'écrire sous la forme $\mathrm{R}^{-(\alpha+\delta)}$, où $\mathrm{R}$ est la rigidité (définie dans l'encadré) $)^{(a)}$ et les coefficients $\alpha$ et $\delta$ sont respectivement liés au spectre à la source et au processus de diffusion dans la Galaxie $^{(\mathrm{b})}$. Cependant, les résultats d'AMS-02 (fig. 3) ont mis au jour la présence d'une brisure dans les spectres des protons et des noyaux d'hélium [5], située à la même rigidité pour les deux espèces! Si l'on considère la forme initiale supposée du spectre en $\mathrm{R}^{-(\alpha+\delta)}$, deux hypothèses émergent pour expliquer ces résultats : l'existence d'un effet de brisure universel dans le spectre source, ou dans la diffusion.

Pour tester ces deux hypothèses, il faut s'appuyer sur les espèces secondaires de rayons cosmiques, comme les noyaux de bore (B), de lithium ( $\mathrm{Li}$ ) et de béryllium (Be). Ces noyaux sont issus des réactions nucléaires entre les rayons cosmiques primaires et le milieu interstellaire, et leur spectre va donc rendre compte d'une "double » diffusion : celle effectuée d'abord par leurs noyaux parents, puis leur propre diffusion. On s'attend donc à une loi de puissance de la forme $\mathrm{R}^{-(\alpha+2 \delta)}$ pour ces espèces. Les choses deviennent maintenant intéressantes si l'on considère un rapport "secondaire sur primaire ", comme le rapport B/C des spectres des noyaux de bore (d'origine secondaire) et de carbone (d'origine primaire). En étudiant un tel rapport, on s'attend à obtenir un spectre de la forme
$\mathrm{R}^{-(\alpha+2 \delta)} / \mathrm{R}^{-(\alpha+\delta)}=\mathrm{R}^{-\delta}$. Ainsi, selon que la brisure spectrale se trouve à la source (indice $\alpha$ ) ou dans la diffusion (indice $\delta$ ), l'impact sera nul sur le rapport B/C dans le premier cas, et visible dans le second cas. Pour trancher la question, il faut attendre des études plus poussées et l'analyse d'autres espèces secondaires comme le lithium (fig. 3) et le béryllium, qui sont en cours dans la collaboration AMS.

\section{À la recherche de la mystérieuse matière noire}

\section{Le principe de détection indirecte}

Depuis les années 1980, la traque de la matière noire dans le rayonnement cosmique est devenu un nouveau moteur de la discipline. La matière noire est observée par ses effets gravitationnels à toutes les échelles [6], et la Voie lactée ne fait pas exception : celle-ci est en effet entourée d'un halo de matière noire d'un rayon d'environ $10^{6}$ années-lumière (soit 20 fois la taille de la Galaxie visible) et de $10^{12}$ masses solaires (soit 20 fois la masse "visible " de la Galaxie).

Dans le cadre de modèles de physique des particules au-delà du modèle standard, la matière noire serait constituée de nouvelles particules, qui pourraient s'annihiler entre elles pour produire notamment des photons, des positons et des antiprotons. L'approche de la détection indirecte de matière noire, qui complémente les expériences de détection directe [7] et sur accélérateur [8], consiste à rechercher un possible excès dans les flux de ces trois espèces qui ont l'avantage d'être rares dans le rayonnement cosmique. La question cruciale est donc celle de la maitrise du calcul des contributions astrophysiques de ces espèces, afin de les distinguer d'une éventuelle contribution de matière noire. En effet, quel que soit le phénomène ayant produit ces particules, toutes vont se propager dans la Galaxie et se mélanger pour donner le spectre final mesuré.

\section{Antiprotons et positons : matière noire ou astrophysique standard?}

Les antiprotons et les positons sont donc des cibles privilégiées pour la recherche indirecte de matière noire. Ce sont des espèces secondaires rares, créées par l'interaction des protons et noyaux d'hélium sur le milieu interstellaire. On observe ainsi autour de $1 \mathrm{GeV}$ des rapports $\mathrm{e}^{+} / \mathrm{p}$ et anti- $\mathrm{p} / \mathrm{p}$ de l'ordre de $10^{-2}$ et $10^{-4}$ respectivement. Expérimentalement, mesurer les flux de positons et d'antiprotons est très difficile à réaliser, car il faut obtenir de bons facteurs de réjection qui est la capacité du détecteur à trouver une aiguille (un antiproton par exemple) dans une meule de foin (les protons). Il s'agit donc d'être rigoureux et prudent dans l'analyse et l'interprétation des données !

Historiquement, ce sont les premières mesures du flux d'antiprotons à la fin des années 1970 qui ont créé l'émoi chez les physiciens : en plus de la composante astrophysique standard, d'origine purement secondaire, une contribution supplémentaire était nécessaire pour ajuster les données. Hélas, les mesures suivantes montrèrent que ce flux avait été surestimé expérimentalement et sous-estimé dans la modélisation $\mathrm{du}$ fond astrophysique. Les énergies des antiprotons détectés par AMS-02 couvrent aujourd'hui la gamme de $1 \mathrm{GeV}$ à $450 \mathrm{GeV}$ avec une précision de l'ordre de quelques pourcents au $\mathrm{GeV}$, augmentant à $30 \%$ aux plus hautes énergies [5]. L'interprétation de ces nouvelles données reste très débattue : les plus enthousiastes pensent avoir vu un excès alors que les plus prudents soulignent que la modélisation $\mathrm{du}$ fond astrophysique reste incomplète. Car, de manière paradoxale, le facteur limitant ne réside maintenant plus dans l'incertitude statistique des données mais bien dans les incertitudes liées aux modèles 


\section{$\gg>$}

de transport dans la Galaxie. En tenant compte de ces incertitudes, il est possible d'évaluer la contribution maximale de matière noire acceptable pour de ne pas être au-dessus des données. Et les résultats sont de très grande portée : s'il est difficile de conclure actuellement sur la nécessité d'une telle contribution, les antiprotons mesurés par AMS-02 et les rayons gamma mesurés par Fermi sont les observables les plus contraignantes aujourd'hui pour la recherche de matière noire.

Mais qu'en est-il des positons ? Ces derniers ont défrayé la chronique à plusieurs reprises ces dernières années, et ont été largement médiatisés. En effet, les résultats de la collaboration PAMELA [9], publiés dans la prestigieuse revue Nature en 2009, ont créé le buzz en mettant en évidence une remontée inexpliquée à haute énergie dans la fraction de positons (rapport du nombre de positons sur le nombre total d'électrons et positons). La collaboration AMS a confirmé l'excès de positons (fig. 4), ceci avec une précision inégalée, et en étendant les mesures à plus haute énergie [5]. L'hypothèse d'une contribution issue de l'annihilation de matière noire a rapidement fait surface, mais est-ce bien plausible ? Malheureusement non, pour plusieurs raisons. Tout d'abord, la production de positons par la matière noire prédite théoriquement est 100 à 1000 fois plus faible que l'excès observé. Par ailleurs, si un tel excès était réellement dû à la matière noire, il aurait été naturel que celle-ci se soit également manifestée pour les antiprotons et les rayons gamma, ce qui n'est pas le cas. Mais l'objection majeure tient au fait que dès les années 80, des physiciens avaient prédit cette remontée en tenant compte d'une source additionnelle de positons, les pulsars (des étoiles à neutrons fortement magnétisées en rotation rapide sur elles-mêmes), et cette explication astrophysique paraît plus « naturelle » que l'hypothèse matière noire.

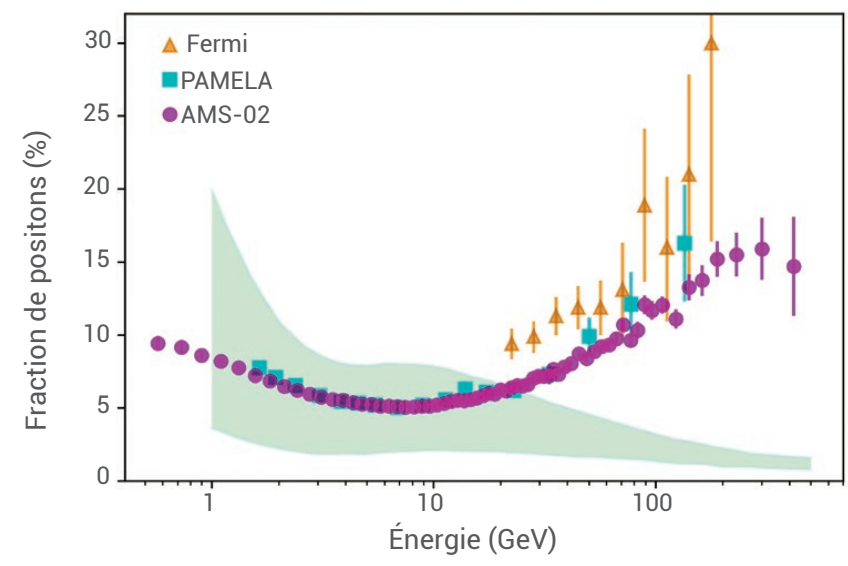

4. " $\mathrm{L}^{\prime}$ excès » de la fraction de positons. Fraction de positons $\mathrm{N}_{e^{+}} /\left(\mathrm{N}_{e^{+}}+\mathrm{N}_{\mathrm{e}^{-}}\right)$en fonction de l'énergie mesurée par AMS-02 (disques violets), comparée aux précédentes mesures de PAMELA (carrés turquoise) et Fermi (triangles orange). La zone vert clair indique la prédiction théorique avec ses incertitudes pour la contribution d'un fond astrophysique purement secondaire, c'est-à-dire d'électrons et de positons uniquement issus de réactions nucléaires avec le milieu interstellaire. L'augmentation observée expérimentalement à haute énergie n'est pas compatible avec cette contribution seule : s'il est tentant de vouloir chercher une explication en termes de matière noire, l'explication la plus naturelle est celle de positons accélérés dans des pulsars proches.

\section{Conclusions}

Construire et installer un détecteur complexe sur la station spatiale internationale est une prouesse technologique. Après six ans de prise de données, les résultats obtenus sont incroyables de précision et feront référence pour plusieurs décennies. La surprise majeure a été l'observation d'une brisure spectrale dans la composante nucléaire du rayonnement cosmique galactique. À ce jour, l'interprétation des mesures d'AMS-02 pour les positons et les antiprotons ne donne pas d'indication claire en faveur de la matière noire. Cependant, la question reste débattue ; la quête de la matière noire continue également avec la recherche d'antideutérons. Par ailleurs, le potentiel d'AMS-02 n'est pas encore complètement exploité, puisque les flux de la plupart des éléments et les rapports isotopiques n'ont pas encore été publiés. Prévu jusqu'en 2024, AMS-02 va continuer de jouer un rôle moteur et fondamental dans la discipline. (a) Dans le domaine du rayonnement cosmique, les flux ne sont pas toujours exprimés en fonction de la même unité d'énergie. Alors qu'aux très hautes énergies les flux sont souvent exprimés en fonction de l'énergie totale (eV ou $\mathrm{GeV})$, quantité mesurée dans les détecteurs, l'énergie cinétique par nucléon $(\mathrm{GeV} / \mathrm{n})$ a été préférée historiquement aux plus basses énergies. En réalité, les processus d'accélération et de diffusion dépendent des champs magnétiques et donc de la charge des particules, et il est plus naturel d'exprimer les processus astrophysiques associés en fonction de la rigidité en gigavolts (GV), quantité qui est aussi reconstruite dans le détecteur AMS-02. A des facteurs numériques près, toutes ces représentations sont équivalentes au-delà de quelques dizaines de $\mathrm{GeV}$.

(b) La théorie de l'accélération de particules dans les ondes de chocs via les mécanismes dits de Fermi [3] prévoit une loi de puissance quasi universelle en $\mathrm{R}^{-\alpha}$, avec $\alpha \sim 2$. D'autre part, les prédictions pour la diffusion dans les champs magnétiques turbulents prévoient une dépendance $\mathrm{R}^{\delta}$, avec $\delta \sim 0,3$ à 0,5 (turbulence de type Kolmogorov ou Kraichnan). Dans l'équation de diffusion qui régit la propagation $\mathrm{du}$ rayonnement cosmique, la solution pour les noyaux est grossièrement donnée par le spectre source divisé par la diffusion, soit une loi de puissance $\mathrm{R}^{-(\alpha+\delta)}$, qui doit atteindre une pente de 2,8 pour reproduire les données (voir fig. 1).
$4 \cdot 0$. Deligny et T. Suomijärvi, « Les rayons cosmiques d'énergie extrême ", Reflets de la physique $\mathbf{4 3}$ (2015) 31-37.

5• AMS collaboration, Phys. Rev. Lett. 113 (2014) 121101 (fraction de positons), 113 (2014) 121102 (flux d'électrons et de positons), 114 (2015) 171103 (flux de protons), 115 (2015) 211101 (flux d'hélium), 117 (2016) 091103 (flux d'antiprotons) et 117 (2016) 231102 (rapport bore/carbone).
6• F. Combes, «La matière noire, une sombre affaire », Reflets de la physique 51 (2016) 4-10.

7• M. Stern, «Edelweiss à la recherche de la matière noire ", Bulletin de la SFP 155 (2006) 15-20.

$8 \cdot A$. Zabi et N. Morange, « Une nouvelle ère pour le LHC ", Reflets de la physique 52 (2017) 28-30.

$9 \cdot 0$. Adriani et al., "An anomalous positron abundance in cosmic rays with energies 1.5-100 GeV", Nature 458 (2009) 607. 